\section{Papilloedema in a Patient with Cervical Dystony - A Case Report}

\author{
Makarov Igor Anatol'evich ${ }^{1,2^{*}}$ \\ 1/nstitute of Biomedical Problems, Moscow, Russia \\ ${ }^{2}$ Research Center of Neurology, Moscow, Russia
}

\begin{abstract}
Cervical (torsion) dystonia or torticollis is disorder characterized by wrong position of the head. It is suspected to be caused by acquired damages in extrapyramidal nervous system and in other cerebral structures leading to unconscious motor function. Neuron disturbances entail hypertonic neck muscles. Among all types of torticollis, ophthalmologists should pay attention to laterocollis manifested through the tilting of the head to one side. A 25-year-old man has been diagnosed with laterocollis for 3 years. The papilledema was more evident with the increased optic nerve disc in the nasal segment, and also optic nerve disc protrusion over the retina. Changes were more evident on the right eye. The temporal sector within the range of $90^{\circ}$ remained intact. The moderate decrease of RNFL thickness was defined in the superior and inferior-nasal segments on the right eye, and the low local decrease of RNFL was found in the nasal segment on the left eye.
\end{abstract}

Keywords: Intracranial pressure; Papilledema; RNFL; Torticollis

\section{Introduction}

Cervical (torsion) dystonia (torticollis) is disorder externally manifested wrong position of the head. This pathology is most common in women and debut aged from 20 to 60 years. It is believed that the reason is due to acquired disorders in extrapyramidal nervous system and other cerebral structures, which are generally responsible for

*Corresponding author: Makarov Igor Anatol'evich, Institute of Biomedical Problems, Moscow, Russia; Research Center of Neurology, Moscow, Russia, Tel: +7 9057067183; E-mail: igor-niigb@yandex.ru

Citation: Makarov IA (2018) Papilledema in Patient with Cervical Dystony - A Case Report. J Ophthalmic Clin Res 5: 40.

Received: November 03, 2017; Accepted: February 26, 2018; Published: March 12, 2018 unconscious motor function. Irregularities in the neurons lead to an increase in muscle tone of the neck. Clinical manifestations can be varied; in the initial stages are imperceptible, movements of the head smooth, only the patient himself can feel the quiver of the neck muscles. However, visually it is already possible to notice some thickening of the muscle, the person experiences pain at palpation. There is involuntary rotation of the head in one direction or another in the disorder progression. The patient cannot self-correct the position of the head because the neck muscles in these cases are not subject to the will of man. Further, in the pathological process engages more muscles, and change in head position occurs not only in one but in two or even in three directions.

There are several forms of the torticollis depending on the position of the head;

Laterocollis: The head bends to the side and the patient touches his ear with his shoulder

Retrocollis: Backward deflection, head thrown

Antecollis: Chin lowered down

Torticollis: Turn of the head, chin in contact with the shoulder

It was found that the most frequent manifestations of cervical dystonia are muscle pains, as well as various emotional disorders of the personality, psychological problems and social limitations. A high prevalence of anxiety and depressive disorders is known in these patients.

The main method of conservative treatment at present is the use of botulinum toxin preparations, which are injected into intense hypertrophied muscles to block neuromuscular transmission. Injections should be repeated at intervals of 3 to 5 months. In recent years, a more effective method of high-frequency pulsed electrostimulation of structures of the extrapyramidal system of the brain by stereotactic operative access has been used [1].

\section{Case Report}

A 25 year old man was admitted to the Research Center of Neurology for the next course of botulinotherapy, with the diagnosis: Cervical dystonia, $3^{\text {rd }}$ degree of severity and laterocollis to the right. The debut was 3 years ago. Injections of botulinum toxin into the affected muscles and massage of the collar zone are performed with periodicity of 4 to 5 months.

\section{Neuro-ophthalmic status}

- Arbitrary eye movements were not observed, the pupil size corresponds to the illumination

- Ptosis of upper eyelid, strabismus, and different width of pupils were absent

- Friendly eye movements were without abnormalities

- Visual acuity was 20/20 without correction

- Intraocular pressure was $12 / 11 \mathrm{mmHg}$ by pneumotonometry 


\section{Computer perimetry}

The vision field was normal, the retina photosensitivity was normal. Relative and absolute cattle and the blind spot were not enlarged. There were not pathological changes in the anterior segment by slit lamp microscopy examination.

Ophthalmoscopy and Optical Coherent Tomography (OCT) of the optic disc (Figures $1 \&$ 2); optic nerve disc was pale pink, borders in the nasal, upper and lower regions were blurred. Disc promised anteriorly, stranded above the level of the retina in these areas. The disc diameter was slightly enlarged in the nasal area. The changes were more pronounced on the right eye. The caliber of blood vessels was increased, the fullness of the veins, the ratio of the artery/vein was $1 / 2$, the tortuosity was moderate, the course of the vessels was normal. The macular area and the retina on both eyes were without features, druses and pathological foci.

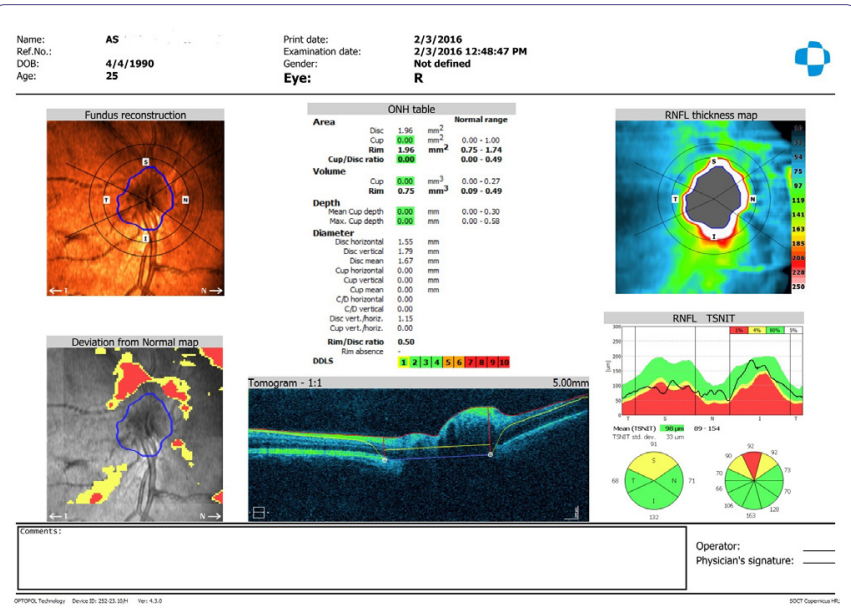

Figure 1: Optical Coherent Tomography (OCT) of the right peripapillary area.

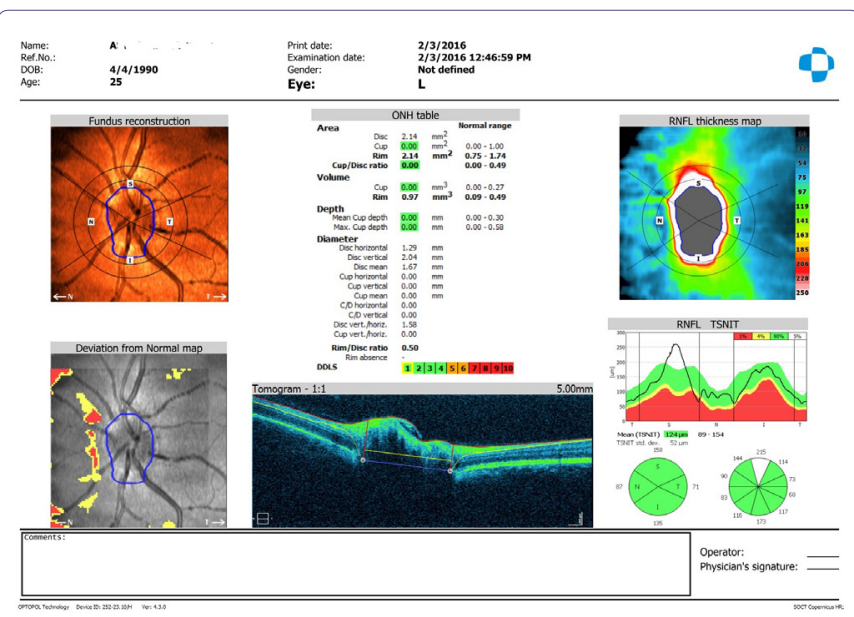

Figure 2: Optical Coherent Tomography (OCT) of the left peripapillary area.

\section{The OCT conclusion}

There were moderate decrease in the thickness of the Retina Nerve
Fibers Layer (RNFL) in the superior and superior-nasal segments on the right eye and there were moderate local decrease of RNFL in the superior-nasal segment on the left eye.

\section{The diagnosis}

Papilledema of the both eyes in the initial stage (by Tron classification) [2] or I stage (by Frisen classification) [3]. RNFL thickness was decreased in the superior, superior-nasal and nasal segments of the optic nerve region. It was recommended by the neurosurgeon aid.

\section{Discussion}

It is commonly known that the brain is separated from the osteocranium with three shells. There is subarachnoid cavity between the vascular and web shells. The brain is supplied with blood through the vessels of the internal carotid and vertebral arteries. Outflow of blood from the brain is carried out through the system of cerebral veins, venous sines and internal jugular veins. Healthy adult person has approximately $150 \mathrm{ml}$ of blood in his vascular system of the brain and $100 \mathrm{ml}$ of which in his venous system. The subarachnoid cavity is bound to ventricles of the brain and filled with the cerebrospinal fluid which is produced constantly, approximately, at the rate of $0.3 \mathrm{ml}$ in $\min$ [4]. Because of the gradient between the intracranial pressure and pressure in the dural venous sinus, the absorption of cerebrospinal fluid is carried out in cover villuses and then its further transformation in the system of venules of the sagittal and sigmoid venous sines. The level of intracranial pressure is variable and depends on position of the body, respiration depth, increasing when coughing, when performing the Valsalva maneuver, when holding breath.

It is known that one of the diagnostic sign of the increased intracranial pressure is edema of the optic nerve disc. The edema is caused by anatomic features of the subarachnoid cavity which stretches to the eye back pole $[5,6]$.

At the same time, the patients with the cervical dystonia have tight and hypertrophied muscles of the neck which are so spastically strained that they arguably strangulate the veins blocking the blood outflow through them. As it was told above, outflow of the blood from the brain is carried out on the jugular veins. The jugular veins pass lateral sides of the neck. Perhaps, at that kind of cervical dystonia when the head is inclined sideways, that is at the laterocollis, the neck muscles spastically strained and strangulate the jugular veins, so as to block the blood outflow. In this regard, there is a local hypervolemia of the blood in dural venous sinuses of the brain. It is not excluded; the rising pressure leads to decrease of the blood pressure gradient between dural sinuses and cerebrospinal fluid in cover villuses of the subarachnoid cavity. Depression of the cerebrospinal fluid absorption in the villuses may be lead to increasing the intracranial pressure. Such intracranial hypertension is not as strongly evident as at total block of cerebrospinal fluid outflow pathways by a tumor or aneurysm. But nevertheless its long existence can lead to papilledema finally.

Shah VA, et al., [7] describe neurological picture of the intracranial hypertension after unilateral neck dissection. Kurschel S, et al., [8] report about a clinical case of existence of papilledema at the 13-yearold boy with torticollis, classifying it as secondary form of the idiopathic intracranial hypertension. Also, the clinical case-report of the papilledema with Arnold Chianti malformation type I was reported [9]. Popiela M, et al., [10] describe the bilateral papilledema at the 
patient with Camurati-Engelmann diseases, specifying about the edema reason following of the jugular veins strangulation.

Long term duration papilledema leads to block of axoplasmatic liquid current in nerve fibers. This can lead to neurodegeneration of nerve cells that was found by decreasing RNFL thickness in OCT investigation at the young man. This decreasing is not so strongly as optic nerve disc atrophy in expressed intracranial pressure.

In this study it is clear that the RNFL thickness decreasing comes not at once, and after several years from debut of laterocollis. This neurodegeneration is not strongly evident as it is about moderate rising of intracranial pressure owing to disturbance the venous outflow of the blood from the brain. In the studies Smith KA, et al., [11] it is established that at $82 \%$ of patients with the idiopathic intracranial hypertension had visual improvement and decreased RNFL thickness and indicators improved after stenting of dural venous sinus.

Obviously general nature of papilledema at laterocollis with the same changes at some astronauts at long duration space flight missions. These changes are described in literature as space-flight associated neuro-ocular syndrome [12]. One of the reasons of the syndrome also consists in the venous blood outflow disturbance on the jugular veins from the brain in the microgravity space flight $[13,14]$. Long observation over patients with similar pathogenesis of papilledema can bring more data for studying the influence of microgravity on the optic nerve.

\section{References}

1. Steeves TD, Day L, Dykeman J, Jette N, Pringsheim T (2012) The prevalence of primary dystonia: a systematic review and meta-analysis. Mov Disord 27: 1789-1796.

2. Frisen L (1982) Swelling of the optic nerve head: a staging scheme. J Neurol Neurosurg Psych 45: 13-18.
3. Tron EG (1955) Diseases of the eye pathway. Medgiz Leningrad USSR (In Russ).

4. Partington T, Farmery A (2014) Intracranial pressure and cerebral blood flow. Anesthesia and Intensive Care Medicine 15: 189-194.

5. Berdahl JP, Yu DY, Morgan WM (2012) The translaminar pressure gradient in sustained zero gravity, idiopathic intracranial hypertension, and glaucoma. Medicine Hypotheses 79: 719-724.

6. Marek B, Harris A, Kanakamedala P, Lee E, Amireskandari A, et al. (2014) Cerebrospinal fluid pressure and glaucoma: Regulation of trans-lamina cribrosa pressure. Br J Ophthalmol 98: 721-725.

7. Shah VA, Yang GS, Smith R, Lee AG (2007) Intracranial hypertension after unilateral neck dissection. J Neuro Neurosurg Psychiatry 78: 403-404.

8. Kurschel S, Maier R, Gellher V, Eder HG (2007) Chiari I malformation and intra-cranial hypertension: a case-based review. Childs Nerv Syst 23. 901-905.

9. Imane M, Asmae M, Tonfik R, Rachid S (2016) Papillary oedema revealing Arnold Chiari malformation type I: about a case. Pan Afr Med J 24: 293.

10. Papiela M, Austin M (2015) Bilateral papilloedema in Camurati-Engelmann diseases. BMJ Case Rep 18: 2015.

11. Smith KA, Peterson JC, Arnold PM, Camarata PJ, Whittaker TJ, et al. (2017) A case series of dural venous sinus stenting in idiopathic intracranial hypertension: association of outcomes with optical coherence tomography. Int J Neurosci 127: 145-153.

12. Lee AG, Mader TH, Gibson CR, Tarver W (2017) Space Flight-Associated Neuro-ocular Syndrome. JAMA Ophthalmol 9: 992-994.

13. Lee AG, Tarver WJ, Mader TH, Gibson CR, Hart SF, et al. (2016) Neuro-Ophthalmology of space flight. J Neuroophthalmol 36: 85-91.

14. Makarov IA, Voronkov YI, Aslanjan MG (2017) Ophthalmic changes associated with long-term exposure to microgravity. Human Physiology 43: 105-113. 